\title{
Bone mineral density, bone remodeling and osteoprotegerin in patients with acute coronary syndrome
}

\author{
Jose Luis Pérez-Castrillon a,*, Laura Abad ${ }^{\mathrm{a}}$, Gemma Vega ${ }^{\mathrm{a}}$, Alberto Sanz-Cantalapiedra ${ }^{\mathrm{a}, \mathrm{b}}$, \\ Angel San Miguel ${ }^{a}$, Angeles Mazón ${ }^{a}$, Daniel De Luis ${ }^{a}$, Antonio Dueñas-Laita ${ }^{a}$ \\ a Department of Internal Medicine, Río Hortega University Hospital, Faculty of Medicine, Cardenal Torquemada s/n, 47010, Valladolid, Spain \\ ${ }^{\mathrm{b}}$ Pediatric Laboratory, Department of Pediatrics-Institute of Biology and Molecular Genetics, Faculty of Medicine, Valladolid, Spain
}

Received 2 April 2007; accepted 23 June 2007

Available online 8 August 2007

\begin{abstract}
The objective of this study was to evaluate the relationship between coronary disease and osteoporosis and determine the effect of osteoprotegerin (OPG) on bone remodeling and bone mineral density (BMD) in a group of patients with acute coronary syndrome. Eightythree patients (52 males and 31 women) with acute coronary syndrome (75 patients with acute myocardial infarction and 8 with unstable angina) with an average age of $61 \pm 10$ years were studied. Levels of osteocalcin, urinarydeoxypyridinoline, OPG and the receptor activator of nuclear factor- $\kappa \mathrm{B}$ ligand (RANKL) were determined during the hospital stay. Femoral neck, trochanter and lumbar spine densitometry was carried out using a DXA densitometer. Thirty percent of patients presented osteoporosis (39\% of females and 26\% of males). Osteoporotic patients were older and had a lower weight and height and elevated serum levels of osteocalcin $(3.6 \pm 2.252 .63$ versus $\pm 1.55, p=0.05)$. Levels of OPG and RANKL were similar in both groups and showed no relationship with BMD. In conclusion, no relationship was observed between the OPG/RANKL system and BMD in these patients.
\end{abstract}

(C) 2007 Published by Elsevier Ireland Ltd.

Keywords: Osteoprotegerin; RANKL; Osteoporosis; Acute coronary syndrome

\section{Dear Editor,}

Clinical studies have found an association between osteoporosis and atherosclerosis [1], with people with reduced bone mass or fracture presenting increased global mortality, specifically cardiovascular mortality. Various mechanisms have been implicated in the possible relationship between reduced bone mineral density and coronary disease. Recently, osteoprotegerin (OPG), a protein that regulates the activity and proliferation of osteoclasts, has been implicated in both processes $[2,3]$. Osteoprotegerin is a soluble glycoprotein that acts as a decoy receptor of the

\footnotetext{
* Corresponding author. Department of Internal Medicine, Río Hortega University Hospital, Faculty of Medicine, Cardenal Torquemada s/n, 47010, Valladolid, Spain. Tel.: +34 983 420400; fax: +34 983331566.

E-mail address: castrv@terra.es (J.L. Pérez-Castrillon).
}

receptor activator of nuclear factor- $\kappa \mathrm{B}$ ligand (RANKL) a key piece in the regulation of osteoclastogenesis [4]. In addition, OPG may intervene in the prevention of cardiovascular disease [3]. The objective this study was to evaluate the relationship between coronary disease and osteoporosis and determine the effect of osteoprotegerin on bone remodeling and bone mineral density (BMD) in a group of patients with acute coronary syndrome.

Consecutive patients admitted to our hospital with a diagnosis of acute coronary syndrome according to European Society of Cardiology criteria were included in the study. Patients were analysed between the third and seventh day of hospital stay. All women were postmenopausal. The study was approved by the hospital ethics committee and all patients gave written informed consent. Blood samples were obtained after $8 \mathrm{~h}$ of fasting. Osteocalcin was measured using chemoimmunoluminiscence and osteoprotegerin and RANKL were measured using a commercial ELISA test. 
Urinary deoxypyridinoline levels were determined by chemoimmunoluminiscence. Densitometric studies were conducted in the lumbar spine (L2-L4) and femoral neck and trochanter using an X-ray densitometer (DXA, Lunar Corporation, Madison, Wisconsin, USA).

Eighty-three patients were included in the study (75 patients with acute myocardial infarction and 8 with unstable angina) with an average age $61 \pm 10$ years. Thirty-nine percent were osteoporotic (39\% of females and $26 \%$ of males). Analysis of the patients according to the presence of osteoporosis showed that osteoporotic patients were older and had a lower weight and height. The only analytical parameter that distinguished them was osteocalcin $(3.6 \pm 2.25 \mathrm{nmol} / 1$ vs $2.63 \pm 1.55 \mathrm{nmol} / \mathrm{l}$, $p<0.05$ ), reflecting an increase in bone turnover, without differences in OPG $(10.8 \pm 3.7 \mathrm{pmol} / 1$ vs $9.7 \pm 3.7 \mathrm{pmol} / \mathrm{l}$, $p>0.05)$ and $\operatorname{RANKL}(0.397 \pm 0.12 \mathrm{pmol} / 1$ vs $0.369 \pm$ $0.12 \mathrm{pmol} / \mathrm{l}, p>0.05)$ being found. However, when the patients were classified in quartiles according to the level of OPG there was a lower prevalence of osteoporosis, $14 \%$, in the first quartile $(\mathrm{OPG}<7.29)$,although the difference was not statistically significant.

Our study found a high prevalence of osteoporosis in patients with acute coronary syndrome, probably due to increased bone turnover as shown by the high levels of osteocalcin. Osteoprotegerin was not found to be a protective element against osteoporosis in these patients. However, low levels of osteoprotegerin tended to be associated with a lower prevalence of osteoporosis. The possible mechanism is by blocking RANKL, which stimulates osteoclastogenesis [4]. The RANKL/OPG system plays an important role in destabilizing atheroma plaque by modulating the liberation of enzymes that degrade the matrix, and has been implicated in the relationship between osteoporosis and atherosclerosis [5]. However, there are few studies evaluating the role of the RANKL/OPG system in osteoporosis associated with acute coronary syndrome. Pennisi et al. [6] found no association between levels of OPG and RANKL and bone mineral density or markers of bone remodeling. Their results are similar to ours.

Osteoprotegerin is elevated in patients with acute myocardial infarction and is related to disease severity, morbidity and mortality [7]. In addition, a direct relationship between OPG and inflammatory markers has been found in this group of patients and OPG has been shown to behave as a marker of the inflammatory response $[3,8]$. In vitro studies have shown that proinflammatory cytokines (TNF- $\alpha$, IL-1 $\beta$, angiotensin II, factor activator of platelet growth) induce the expression of
OPG in vascular smooth muscle cells [9]. In addition, the cell necrosis produced during myocardial infarcts would facilitate the release of OPG to the bloodstream, raising its concentration. Proinflammatory cytokines produce increased bone turnover [10] that may be responsible for the reduced bone mass in these patients. The higher levels of osteocalcin, a marker of bone turnover, presented by our coronary patients with osteoporosis would support this hypothesis. However, Farhat et al. [11] found that although levels of TNF- $\alpha$ and IL- 6 were higher in patients with cardiovascular disease than in controls, there was no association with bone mineral density in any site.

In conclusion, osteoprotegerin does not relate to bone mass in patients with acute coronary syndrome and is probably a marker of the inflammatory response.

\section{References}

[1] Tanko LB, Christiansen C, Cox DA, Geiger MJ, McNabb MA, Cummings SR. Relationship between osteoporosis and cardiovascular disease in postmenopausal women. J Bone Miner Res 2005;20:1912-20.

[2] Schoppet M, Preissner KT, Hofbauer LC. RANK ligand and osteoprotegerin: paracrine regulators of bone metabolism and vascular function. Arterioscler Thromb Vasc Biol 2002;22:549-53.

[3] Hofbauer LC, Schoppet M. Clinical implications of the osteoprotegerin/RANKL/RANK system for bone and vascular diseases. JAMA 2004;292:490-5.

[4] Khosla S. Minireview: the OPG/RANKL/RANK system. Endocrinology 2001;142:5050-5.

[5] Sandberg WJ, Yndestad A, Oie E, et al. Enhanced T-cell expression of RANK ligand in acute coronary syndrome. Arterioscler Thromb Vasc Biol 2006;26:857-63.

[6] Pennisi P, Signorelli SS, Riccobene S, et al. Low bone density and abnormal bone turnover in patients with atherosclerosis of peripheral vessels. Osteoporosis Int 2004;15:389-95.

[7] Jono S, Ikari Y, Shioi A, et al. Serum osteoprotegerin levels are associated with the presence and severity of coronary artery disease. Circulation 2002;106:1192-4.

[8] Libby P, Ridker PM, Maseri A. Inflammation and atherosclerosis. Circulation 2002;105:1135-43.

[9] Collin-Osbody P, Rothe L, Anderson F, Nelson M, Maloney W, Osbody P. Receptor activator of NF-kB and osteoprotegerin expression by human microvascular endothelial cells, regulation by inflammatory cytokines and role in human osteoclastogenesis. J Biol Chem 2001;276:20659-72.

[10] Boyle WJ, Simonet WS, Lacey DL. Osteoclast differentiation and activation. Nature 2003;423:337-42.

[11] Farhat GN, Strotmeyer ES, Newman AB, et al. Volumetric and areal bone mineral density measures are associated with cardiovascular disease in older men and women: the health, aging, and body composition study. Calcif Tissue Int 2006;79:102-11. 